\title{
Theory for the coupling between longitudinal phonons and intrinsic Josephson oscillations in layered superconductors
}

\author{
Ch. Helm, Ch. Preis, Ch. Walter, and J. Keller \\ Institut für Theoretische Physik, Universität Regensburg, \\ D-93040 Regensburg, Germany \\ (submitted to Phys.Rev.B)
}

In this publication a microscopic theory for the coupling of intrinsic Josephson oscillations in layered superconductors with longitudinal c-axis-phonons is developed. It is shown that the influence of lattice vibrations on the $c$-axis transport can be fully described by introducing an effective longitudinal dielectric function $\epsilon_{\mathrm{ph}}^{L}(\omega)$. Resonances in the $I$ - $V$-characteristic appear at van Hove singularities of both acoustical and optical longitudinal phonon branches. This provides a natural explanation of the recently discovered subgap structures in the $I$ - $V$-characteristic of highly anisotropic cuprate superconductors. The effect of the phonon dispersion on the damping of these resonances and the coupling of Josephson oscillations in different resistive junctions due to phonons are discussed in detail.

$74.80 \mathrm{Dm}, 74.50+\mathrm{r}, 74.25 \mathrm{Kc}, 74.25 \mathrm{Jb}$

\section{INTRODUCTION}

The $c$-axis transport in the highly anisotropic cuprate superconductors $\mathrm{Tl}_{2} \mathrm{Ba}_{2} \mathrm{Ca}_{2} \mathrm{Cu}_{3} \mathrm{O}_{10+\delta}$ (TBCCO) and $\mathrm{Bi}_{2} \mathrm{Sr}_{2} \mathrm{CaCu}_{2} \mathrm{O}_{8+\delta}$ (BSCCO) can well be described by a stack of Josephson junctions between the superconducting $\mathrm{CuO}_{2}$-multi-layers. This intrinsic Josephson effect can be seen in the multi-branch structure of the $I-V$ characteristic, each branch corresponding to awell-defined number of junctions in the resistive state. 1 2 The intrinsic Josephson effect is observed also in the behaviour of the material in external magnetic fields and under microwave irradiation 1 ,

Recently subgap structures in the $I$ - $V$-characteristic have been discovered as intrinsic properties of the material, 5 which could be explained by the coupling betwen the intrinsic Josephson oscillations and phonons.6 8 This interaction is mediated by the oscillating electric field in the Josephson junction, which excites vibrations of charged ions in the material. In our previous investigations 6 we used a simple model of a system of damped harmonic oscillators in order to describe the dynamics of oscillating ions in the barrier. We were able to derive an analytic expression for the dc-current density $j(V)$ as function of the dc-voltage $V$ for one resistive junction

$$
j(V)=j_{q p}(V)+\frac{j_{c}}{2} \frac{\omega_{p}^{2}}{\omega^{2}} \frac{\epsilon_{2}+\frac{\sigma}{\omega \epsilon_{0}}}{\epsilon_{1}^{2}+\left(\epsilon_{2}+\frac{\sigma}{\omega \epsilon_{0}}\right)^{2}} .
$$

where the voltage $V$ is related to the Josephson oscillation frequency $\omega$ by $V=\hbar \omega /(2 e)$ and $\epsilon(\omega)=\epsilon_{1}(\omega)+$ $i \epsilon_{2}(\omega)$ is the dielectric function of the oscillating ions. From this result it can be seen that the $I$ - $V$-curve has a maximum at the frequency (voltage) where the real part $\epsilon_{1}$ of the phonon-dielectric function vanishes, which corresponds to a longitudinal eigenfrequency of the phonon system.

With appropriate values for the Josephson plasma freqency $\omega_{p}$, the quasiparticle conductivity $\sigma$, the critical current denstiy $j_{c}$, and the frequencies, dampings and oscillator strengths of phonons in the dielectric function we were able to fit the experimental results for the subgap structures in the $I$ - $V$-curves perfectly. In addition to this, the identification of the maxima of the structure with phonon frequencies provides a natural explanation, why the position of the resonances is completely independent of temperature, magnetic field and the geometry of the probe. The voltage positions of these resonances fan also be related to structures in optical experiments 10 in particular to the reflectivity for oblique incidence 11 .

Despite the clear evidence of the effect a general theory for the coupling between Josephson oscillations and phonons is still missing and shall be presented in the following. In particular, in Refs. 6. 6 the phonon frequencies and the oscillator strengths in the dielectric function were not specified. The clarification of this point and the extension to a general lattice dynamical model including acoustical and optical branches will be one of the main topics of the following investigation.

Another topic concerns the coupling of Josephson oscillations in different resistive junctions. We will show how phonons lead to a coupling of the phases of Josephson oscillations in different resistive junctions. This will be discussed in detail for two resistive junctions and general results will be given for large stacks of resistive junctions. A phase-locking in a stack of Josephson junctions is important for applications of such systems for highfrequency mixers and detectors.

The excitation of phonons by Josephson oscillations in conventional single Josephson junctions has been observed already a long time ago.12 Also in the $I-K$ curves of break-junctions of cuprate-superconductors 3 structures due to phonons might have been identified. The physical mechanism described here can also be applied to these cases but our formalism is particularly suited to treat stacks of Josephson junctions with phonons in the frequency range between the Josephson plasma frequency 
and the gap-frequency.

It is not our intention to explain the details of the $I-V$-characteristic of TBCCO and BSCCO by a realistic lattice dynamical calculation. This is impossible at the moment for the complicated anisotropic superconductors with variable doping showing this effect. Furthermore this would require a detailed theory of superconductivity and the Josephson effect in two- and threelayer systems including information about the superconducting bands, charge distribution, and charge susceptibility between the layers and inside the $\mathrm{CuO}_{2}$ planes which is not available yet. Therefore we discuss a simple model system with superconducting mono-layers where the conduction electron charge is distributed homogeneously along the layers, and a lattice dynamical model with only two phonon bands showing already the basic features of a full theory which will be expected also for realistic systems. We start the discussion with a definition of our model and a short derivation of the basic Josephson equations for a stack of Josephson junctions.

\section{JOSEPHSON EQUATIONS FOR A STACK OF JUNCTIONS}

We consider a system of $N$ superconducting layers separated by insulating barrier material of thickness $d$ forming a stack of Josephson junctions. We treat the superconducting layers as homogeneous metal sheets with a uniform electron distribution along the layers. In this paper we treat only the case of a uniform tunneling current with a constant bias-current and neglect magnetic field effects due to the current flow. In this case all quantities are constant along the layers. Such an approximation is reasonable for a stack of junctions which is short with respect to the Josephson penetration length but long enough to neglect finite size effects in the ionic polarisation.

The tunneling current density $j_{n}$ from layer $n$ to $n+1$ creates (two-dimensional) charge density fluctuations $\delta \rho_{n}$ on the layers related by the continuity equation

$$
j_{n}(t)-j_{n-1}(t)=-\delta \dot{\rho}_{n}(t) .
$$

These charge fluctuations create electric fields $E_{n}^{\rho}(t)$ (in the c-direction) in the barrier between layer $n$ and $n+1$ which are constant inside each barrier and are related to the charge fluctuations by the Maxwell equation:

$$
\delta \rho_{n}(t)=\epsilon_{0}\left(E_{n}^{\rho}(t)-E_{n-1}^{\rho}(t)\right)
$$

or

$$
E_{n}^{\rho}(t)=\frac{1}{2 \epsilon_{0}}\left(\sum_{n^{\prime} \leq n} \delta \rho_{n^{\prime}}(t)-\sum_{n^{\prime}>n} \delta \rho_{n^{\prime}}(t)\right) .
$$

Assuming that the current density $j_{n}$ for the first and last barrier is fixed by the bias current density $j$, then with help of Eqs. (2,3) the tunneling current density $j_{n}$ in all the other junctions is related to the bias current density $j$ by

$$
j=j_{n}(t)+\epsilon_{0} \dot{E}_{n}^{\rho}(t) .
$$

The last term is the displacement current density related to the charge fluctuations on the layers. In the following we denote this term by $\dot{D}_{n}(t):=\epsilon_{0} \dot{E}_{n}^{\rho}(t)$ in order to relate the present results to the usual notation of the resistively shunted Josephson junction (RSJ) model, however, one should keep in mind the microscopic origin of this term.

In the following we approximate the tunneling current by a superposition of a Josephson supercurrent density and a quasiparticle current density. Then we have for each junction:

$$
j=j_{c} \sin \gamma_{n}(t)+j_{\mathrm{qp}}\left(E_{n}(t)\right)+\dot{D}_{n}(t) .
$$

The Josephson current density $j_{c} \sin \gamma_{n}(t)$ depends on the gauge invariant phase difference $\gamma_{n}(t)$ between layers $n$ and $n+1$ at positions $z_{n}$ and $z_{n+1}$. It is related to the average total electric field in the barrier

$$
E_{n}(t):=\frac{1}{d} \int_{z_{n}}^{z_{n+1}} E_{z}(z, t) d z
$$

by the second Josephson equation

$$
\frac{\hbar}{2 e d} \dot{\gamma}_{n}(t)=E_{n}(t)
$$

Here small corrections to Eq. (8) for layered superconductors which are discussed in Refs. 1415 are neglected. For the quasi-particle current density we will use in the following an ohmic form $j_{\text {qp }}=\sigma E_{n}$ with a constant conductivity $\sigma$. The generalisation to more realistic forms is straightforward.

The crucial point where the phonons come into play is the relation between the field $D_{n}=\epsilon_{0} E_{n}^{\rho}$, which is created by the charge fluctuations on the superconducting layers alone, and the average electric field Eq. (7) $E_{n}=E_{n}^{\rho}+E_{n}^{\text {ion }}$ which contains also the averaged field $E_{n}^{\text {ion }}$ created by the ionic displacements in the barrier. This will be discussed in detail below.

Before we do this let us summarize the most important parameters which characterize the Josephson system: The first one is the (bare) Josephson plasma frequency $\omega_{p}$ defined by

$$
\omega_{p}^{2}:=\frac{2 e d j_{c}}{\hbar \epsilon_{0}} .
$$

The second one is the so-called characteristic frequency defined by

$$
\omega_{c}:=\frac{2 e V_{c}}{\hbar} .
$$


Here $V_{c}$ is the voltage where the quasiparticle current density equals the value $j_{c}$. It is of the order of the superconducting energy gap and is a measure of the dissipative properties of the junction. In our simple model with a constant conductivity we have $\omega_{c}=2 e d j_{c} /(\hbar \sigma)$. The ratio $\beta_{c}=\omega_{c}^{2} / \omega_{p}^{2}$ is the McCumber parameter, which is $\beta_{c} \gg 1$ for the strongly anisotropic cupratesuperconductors. Moreover, for these materials there exist phonons with frequencies in the range $\omega_{p}<\omega_{\text {phon }} \ll$ $\omega_{c}$.

Typically the time-dependence of the phase difference $\gamma(t)$ can be written in the so-called resistive state as

$$
\gamma(t)=\theta+\omega t+\delta \gamma(t)
$$

where $\omega=\langle\dot{\gamma}\rangle$ is the time-average of the phase velocity which is non-zero for a junction in the resistive state. It determines the dc-voltage $V=\langle E\rangle d=\hbar\langle\dot{\gamma}\rangle /(2 e)$ across the junction. In the asymptotically stable state and for large values of the McCumber parameter $\beta_{c}$ the oscillating part $\delta \gamma(t)$ is small and oscillates with the frequency $\omega$.

\section{EXCITATION OF PHONONS BY JOSEPHSON OSCILLATIONS}

Now let us turn to the discussion of lattice vibrations. Quite generally the lattice displacement of an ion of type $\kappa$ with mass $M_{\kappa}$, charge $Z_{\kappa}$ in unit cell $l$ is determined by the following equation of motion

$$
M_{\kappa} \ddot{u}_{\alpha}\left(\begin{array}{l}
l \\
\kappa
\end{array}\right)+\sum_{l^{\prime} \kappa^{\prime}} \Phi_{\alpha \beta}\left(\begin{array}{ll}
l & l^{\prime} \\
\kappa & \kappa^{\prime}
\end{array}\right) u_{\beta}\left(\begin{array}{l}
l^{\prime} \\
\kappa^{\prime}
\end{array} \mid t\right)=Z_{\kappa} E_{\alpha}^{\rho}\left(\begin{array}{l}
l \\
\kappa
\end{array} \mid t\right) .
$$

Here $E_{\alpha}^{\rho}\left(\begin{array}{l}l \\ \kappa\end{array} \mid t\right)$ is the local driving field at the equilibrium position $\vec{R}\left(\begin{array}{l}l \\ \kappa\end{array}\right)=\vec{R}(l)+\vec{R}(\kappa)$ of the ion generated by the charge fluctuations $\delta \rho_{n}(t)$ on the superconducting layers. Note that the vibrating ions may be both in the barrier material and on the superconducting layers. The superconducting electrons are assumed to move together with the ions of the layers.

While the RSJ-equations for the phases are highly nonlinear, the relations between lattice displacements and electric fields are linear, consequently we may analyse the response for each frequency $\omega$ separately. With a harmonic ansatz of the form $u_{\alpha}\left(\begin{array}{l}l \\ \kappa\end{array} \mid t\right)=u_{\alpha}\left(\begin{array}{l}l \\ \kappa\end{array}\right) e^{-i \omega t}$ we obtain for the amplitude

$$
\begin{aligned}
u_{\alpha}\left(\begin{array}{l}
l \\
\kappa
\end{array}\right) & =\frac{1}{N} \sum_{\vec{q} \lambda} \sum_{l^{\prime} \kappa^{\prime} \beta} \frac{e_{\alpha}(\kappa \mid \vec{q} \lambda) e_{\beta}^{*}\left(\kappa^{\prime} \mid \vec{q} \lambda\right)}{\omega^{2}(\vec{q} \lambda)-\omega^{2}} \\
& \times \frac{e^{i \vec{q}\left(\vec{R}(l)-\vec{R}\left(l^{\prime}\right)\right)}}{\sqrt{M_{\kappa} M_{\kappa^{\prime}}}} e Z_{\kappa^{\prime}} E_{\beta}^{\rho}\left(\begin{array}{l}
l^{\prime} \\
\kappa^{\prime}
\end{array}\right) .
\end{aligned}
$$

Here $\omega^{2}(\vec{q} \lambda)$ and $\vec{e}(\kappa \mid \vec{q} \lambda)$ are the eigenvalues and eigenvectors of the dynamical matrix

$$
\sum_{\kappa^{\prime} \beta} D_{\alpha \beta}\left(\begin{array}{c}
\vec{q} \\
\kappa \kappa^{\prime}
\end{array}\right) e_{\beta}\left(\kappa^{\prime} \mid \vec{q} \lambda\right)=\omega^{2}(\vec{q} \lambda) e_{\alpha}(\kappa \mid \vec{q} \lambda)
$$

defined by

$$
D_{\alpha \beta}\left(\begin{array}{c}
\vec{q} \\
\kappa \kappa^{\prime}
\end{array}\right)=\sum_{l^{\prime}} \frac{1}{\sqrt{M_{\kappa} M_{\kappa^{\prime}}}} \Phi_{\alpha \beta}\left(\begin{array}{cc}
l & l^{\prime} \\
\kappa & \kappa^{\prime}
\end{array}\right) e^{i \vec{q}\left(\vec{R}\left(l^{\prime}\right)-\vec{R}(l)\right)} .
$$

The force-constant matrix contains the quantummechanical short-range interactions but also the shortrange and long-range Coulomb interactions (the latter being of the form $\sim q_{\alpha} q_{\beta} / q^{2}$ ) between the ions, but not the fields set-up by the conduction electrons on the layers. The eigenfrequencies $\omega(\vec{q} \lambda)$ are therefore by construction the bare phonon frequencies in the absence of the conduction electron charge fluctuations $\delta \rho_{n}(t)$. They include possible renormalisation by intra-atomic electronic polarisation.

For the further discussion it is convenient to label the lattice dynamical unit cells by $l=\left(l_{x}, l_{y}, l_{z}\right)$ with $l_{z}=n$ denoting the superconducting layer in which the lattice cell is contained (see Fig. 1). Then the zcomponent of the position vector $\vec{R}\left(\begin{array}{c}l \\ \kappa\end{array}\right)$ for $l=\left(l_{x}, l_{y}, n\right)$ does not depend on $l_{\|}=\left(l_{x}, l_{y}\right)$ and we may write $R_{z}\left(\begin{array}{c}l \\ \kappa\end{array}\right)=R_{z}\left(\begin{array}{c}n \\ \kappa\end{array}\right)=R_{z}(n)+R_{z}(\kappa)$. Furthermore the origin of the unit-cell may be chosen on the superconducting layer, so that $R_{z}(n)=z_{n}$.

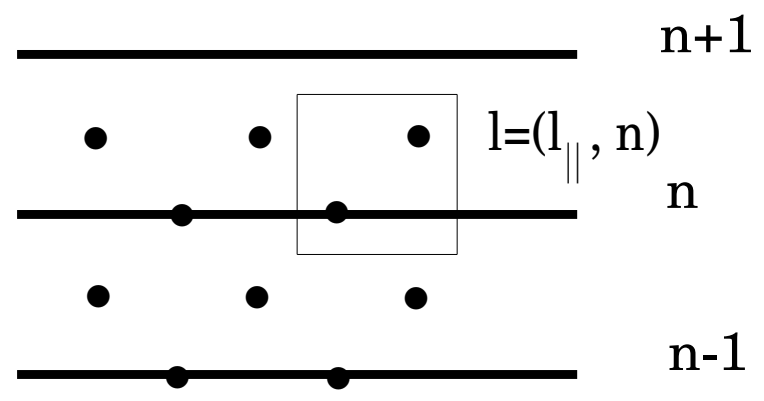

FIG. 1. Labeling of unit cells.

In our model for the superconductivity in the layers we have to assume that the mobile electronic charge is spread out uniformly along the layers, because a microscopic theory connecting the superconducting bands and the atomic sites in the $\mathrm{CuO}_{2}$-layers is still missing. Therefore the field $E_{\beta}^{\rho}\left(\begin{array}{l}l \\ \kappa\end{array}\right)$ does not depend on the position $x$ along the layer and has only a z-component. In Eq. (13) only modes with $q_{\|}=0$ appear and we may write for the displacement amplitude in z-direction of an ion of type $\kappa$ in barrier $n$ :

$$
\begin{aligned}
u_{z}\left(\begin{array}{c}
n \\
\kappa
\end{array}\right) & =\frac{1}{N_{z}} \sum_{q_{z} \lambda} \sum_{n^{\prime} \kappa^{\prime}} \frac{e_{z}\left(\kappa \mid q_{z} \lambda\right) e_{z}^{*}\left(\kappa^{\prime} \mid q_{z} \lambda\right)}{\omega^{2}\left(q_{z} \lambda\right)-\omega^{2}} \\
& \times \frac{e^{i q_{z}\left(z_{n}-z_{n^{\prime}}\right)}}{\sqrt{M_{\kappa} M_{\kappa^{\prime}}}} e Z_{\kappa^{\prime}} E_{z}^{\rho}\left(\begin{array}{c}
n^{\prime} \\
\kappa^{\prime}
\end{array}\right) .
\end{aligned}
$$

Now let us specify the driving field $E_{z}^{\rho}\left(\begin{array}{c}n \\ \kappa\end{array}\right)$ for the ions in more detail: Summing up the electric fields created by the charge fluctuations on the different layers we find: 


$$
E_{z}^{\rho}\left(\begin{array}{ll}
n \\
\kappa
\end{array}\right)= \begin{cases}\frac{1}{2 \epsilon_{0}}\left(\sum_{n^{\prime} \leq n} \delta \rho_{n^{\prime}}-\sum_{n^{\prime}>n} \delta \rho_{n^{\prime}}\right) & \text { for } \kappa \in \text { barrier } \\
\frac{1}{2 \epsilon_{0}}\left(\sum_{n^{\prime}<n} \delta \rho_{n^{\prime}}-\sum_{n^{\prime}>n} \delta \rho_{n^{\prime}}\right) & \text { for } \kappa \in \text { layer. }\end{cases}
$$

Note that for ions inside the barrier the driving field does not depend on the position $R_{z}(\kappa)$ of the ion and is equal to the constant field $E_{n}^{\rho}$ introduced in Eq. (任):

$$
E_{z}^{\rho}\left(\begin{array}{l}
n \\
\kappa
\end{array}\right)= \begin{cases}E_{n}^{\rho} & \text { for } \kappa \in \text { barrier } \\
\frac{1}{2}\left(E_{n}^{\rho}+E_{n-1}^{\rho}\right) & \text { for } \kappa \in \text { layer. }\end{cases}
$$

In order to find a relation between the lattice displacements and the driving field we introduce the Fourier transformation

$$
E^{\rho}\left(q_{z}\right)=\sum_{n} E_{n}^{\rho} e^{-i q_{z} z_{n}}
$$

Then

$$
\sum_{n} Z_{\kappa} E_{z}^{\rho}\left(\begin{array}{l}
n \\
\kappa
\end{array}\right) e^{-i q_{z} z_{n}}=: \tilde{Z}_{\kappa}^{*}\left(q_{z}\right) E^{\rho}\left(q_{z}\right)
$$

with the $q_{z}$-dependent effective charge

$$
\tilde{Z}_{\kappa}\left(q_{z}\right)= \begin{cases}Z_{\kappa} & \text { for } \kappa \in \text { barrier } \\ \frac{1}{2} Z_{\kappa}\left(1+e^{i q_{z} d}\right) & \text { for } \kappa \in \text { layer }\end{cases}
$$

and consequently

$$
\begin{aligned}
u_{z}\left(\begin{array}{l}
n \\
\kappa
\end{array}\right) & =\frac{1}{N_{z}} \sum_{q_{z} \lambda} \sum_{\kappa^{\prime}} e^{i q_{z} R_{z}\left(\begin{array}{l}
n \\
\kappa
\end{array}\right)} \\
& \times \frac{1}{\sqrt{M_{\kappa}}} \frac{e_{z}\left(\kappa \mid q_{z} \lambda\right) e_{z}^{*}\left(\kappa^{\prime} \mid q_{z} \lambda\right)}{\omega^{2}\left(q_{z} \lambda\right)-\omega^{2}} \frac{e \tilde{Z}_{\kappa^{\prime}}^{*}\left(q_{z}\right)}{\sqrt{M_{\kappa^{\prime}}}} E^{\rho}\left(q_{z}\right) .
\end{aligned}
$$

Thereby the vanishing of $\tilde{Z}_{\kappa}$ for $q_{z} d=\pi$ and for ions on the layers reflects the fact that for alternating electric fields in the barriers no net force is acting on the superconducting layers.

Next we want to relate the driving field to the average total electric field $E_{n}$ in the barrier because this field is connected with the phase difference $\gamma_{n}$ by the second Josephson-equation (8). The microscopic total electric field $E_{z}(x, z)$ is the sum of the fields set-up by both the charge-fluctuations and the displaced ionic charges: $E_{z}(x, z)=E_{z}^{\rho}(x, z)+E_{z}^{\text {ion }}(x, z)$. As $E_{z}^{\rho}(x, z)$ is assumed to be constant inside the barrier and independent of $\mathrm{x}$ we may replace the other two fields also by their averages across the barrier and over one unit cell along the layer. For the averaged fields we have then the relation:

$$
E_{n}^{\rho}=E_{n}-E_{n}^{\text {ion }} .
$$

The field $E_{n}^{\text {ion }}$ in the barrier can be calculated from the difference of the scalar potentials on layers $n$ and $n+1$ produced by the ionic displacements averaged over the area of one unit-cell as

$$
\begin{aligned}
E_{n}^{\text {ion }}=-\frac{e}{\epsilon_{0} v_{c}} & \left(\sum_{\kappa \in \text { barrier }} Z_{\kappa} u_{z}\left(\begin{array}{c}
n \\
\kappa
\end{array}\right)\right. \\
& \left.+\frac{1}{2} \sum_{\kappa \in \text { layer }} Z_{\kappa}\left(u_{z}\left(\begin{array}{c}
n \\
\kappa
\end{array}\right)+u_{z}\left(\begin{array}{c}
n+1 \\
\kappa
\end{array}\right)\right)\right) .
\end{aligned}
$$

Here $v_{c}$ is the volume of one unit cell. The factor $1 / 2$ results from the fact that displacements of ions (with $q_{\|}=0$ ) on a layer do not contribute to the potential on the same layer. Note that this field for $q_{\|}=0$ does not depend on the ionic displacements in other barriers and is closely related to the ionic polarisation in the same barrier. Defining a generalised polarisation by

$$
E_{n}^{\text {ion }}=:-P_{n} / \epsilon_{0},
$$

we may write for the displacement in the barrier

$$
D_{n}:=\epsilon_{0} E_{n}^{\rho}=\epsilon_{0} E_{n}+P_{n},
$$

which has the usual form as in the macroscopic Maxwell theory.

Going over to a Fourier transformation the relation

$$
P\left(q_{z}\right)=\chi\left(q_{z}, \omega\right) \epsilon_{0} E^{\rho}\left(q_{z}\right)
$$

between the polarisation and the driving field is obtained with

$$
\chi\left(q_{z}, \omega\right)=\sum_{\lambda} \frac{\left|\Omega\left(q_{z} \lambda\right)\right|^{2}}{\omega^{2}\left(q_{z} \lambda\right)-\omega^{2}}
$$

and the oscillator strength

$$
\left|\Omega\left(q_{z} \lambda\right)\right|^{2}=\frac{e^{2}}{v_{c} \epsilon_{0}} \sum_{\kappa \kappa^{\prime}} \tilde{Z}_{\kappa}\left(q_{z}\right) \frac{e_{z}\left(\kappa \mid q_{z} \lambda\right) e_{z}^{*}\left(\kappa^{\prime} \mid q_{z} \lambda\right)}{\sqrt{M_{\kappa} M_{\kappa^{\prime}}}} \tilde{Z}_{\kappa^{\prime}}^{*}\left(q_{z}\right) .
$$

The special combination of phase factors contained in $\tilde{Z}_{\kappa}\left(q_{z}\right)$ Eq. 21) are a consequence of the different contribution of ions on and between the superconducting layers to the electric field in the barrier.

Using $\epsilon_{0} E^{\rho}\left(q_{z}\right)=\epsilon_{0} E\left(q_{z}\right)+P\left(q_{z}\right)$ we can solve for $P\left(q_{z}\right)$ :

$$
P\left(q_{z}\right)=\frac{\chi\left(q_{z}, \omega\right)}{1-\chi\left(q_{z}, \omega\right)} \epsilon_{0} E\left(q_{z}\right) .
$$

The relation $D\left(q_{z}\right)=\epsilon_{0} \epsilon_{\mathrm{ph}}^{L}\left(q_{z}, \omega\right) E\left(q_{z}\right)$ defines an effective longitudinal dielectric function

$$
\epsilon_{\mathrm{ph}}^{L}\left(q_{z}, \omega\right)=\frac{1}{1-\chi\left(q_{z}, \omega\right)}
$$

This function has zeros at the eigenfrequencies $\omega\left(q_{z} \lambda\right)$ of the dynamical matrix. Due to the form of the oscillator strengths Eq. (29) only modes with polarization in the cdirection contribute. As the electric field $E_{n}$ is constant along the layers we have $\vec{q}_{\|}=0$ and only longitudinal modes in the c-direction couple, therefore the zeros of 
$\epsilon_{\mathrm{ph}}^{L}\left(q_{z}, \omega\right)$ are exactly at the longitudinal eigenfrequencies of the dynamical matrix.

In the case of a single dispersionless phonon-mode with frequency $\omega_{L}$ the function $\epsilon_{\mathrm{ph}}^{L}(\omega)$ can be directly compared with the dielectric function used in Ref. 6. In fact, in this case $\epsilon_{\mathrm{ph}}^{L}(\omega)$ can be written as

$$
\epsilon_{\mathrm{ph}}^{L}(\omega)=1+\frac{\Omega^{2}}{\omega_{L}^{2}-\Omega^{2}-\omega^{2}} .
$$

The form of the longitudinal dielectric function $\left.\epsilon_{\mathrm{ph}}^{L}\left(q_{z}, \omega\right)=\epsilon_{\mathrm{ph}}^{z z}\left(q_{z}, \omega\right)\right)$ Eq. (31), which we have introduced here, is different from the transverse dielectric function $\left.\epsilon_{\mathrm{ph}}^{T}\left(q_{x}, \omega\right)=\epsilon_{\mathrm{ph}}^{z z}\left(q_{x}, \omega\right)\right)=1+\chi\left(q_{x}, \omega\right)$ In both functions different eigenfrequencies and oscillator strengths enter, however in the limit $q_{z} \rightarrow 0, q_{x} \rightarrow 0$, which is only relevant in optical experiments, the values of both functions are equal.

Finally a comparison of our theory with theoretical investigations in Ref. 16 are in order. In principle there are two different electron-phonon coupling mechanisms, which may couple Josephson oscillations and phonons: 1. the electromagnetic interaction between the ionic charges and the charges of conduction electrons, 2. the dependence of the tunneling matrix element on lattice displacements. The first mechanism is considered in our work, the second in Ref. 16. Both mechanisms require a different theoretical treatment (on a diagrammatical basis the two mechanisms would correspond to different diagrams). It has been argued in Ref. 17 that in the layered cuprate superconductors the charges of the ions in the insulating barrier between superconducting layers are unscreened and therefore have a strong interaction with conduction electrons in the $\mathrm{CuO}_{2}$-layers. We therefore considered this mechanism for our treatment of the coupling between Josephson oscillations and phonons. Though we did not write down a Hamiltonian for the interacting system our method nevertheless is a full microscopic theory which treats the electron-phonon interaction on a random-phase-type level by describing the interaction with the help of internal fields. This approximation is sufficient as long as we do not want to consider the electron-phonon interaction inside the superconducting layers and treat exchange effects between different superconducting layers.

\section{INFLUENCE OF PHONONS ON THE IV-CHARACTERISTIC}

According to the RSJ-like model derived in Eq. (6) the current density in junction $n$ is

$$
j=j_{c} \sin \gamma_{n}(t)+\sigma E_{n}(t)+\dot{D}_{n}(t)
$$

where $D_{n}(t)=\epsilon_{0} E_{n}^{\rho}(t)$ is the electric field in junction $n$ set-up by the charge fluctuations of conduction electrons. As pointed out in the previous section this field can be expressed by the average electric field in the barrier and the generalized polarisation Eq. (25) as $D_{n}(t)=\epsilon_{0} E_{n}(t)+P_{n}(t)$. The polarisation has to be calculated self-consistently from the ionic displacements and depends linearly on the electric field.

Let us discuss first the case of one resistive junction at $n=0$ in the middle of a large stack while all other junctions $n \neq 0$ are in the superconducting state. Then as mentioned previously all the oscillations are governed by one frequency $\omega$, and we can write for the phase for $n=0$ :

$$
\gamma_{0}(t)=\theta_{0}+\omega t+\delta \gamma_{0}(t)
$$

while for $n \neq 0$ we have

$$
\gamma_{n}(t)=\theta_{n}+\delta \gamma_{n}(t)
$$

In the stationary state $\delta \gamma_{n}(t)$ oscillates with the same frequency $\omega$,

$$
\delta \gamma_{n}(t)=\delta \gamma_{n} e^{-i \omega t}+c . c .
$$

Higher harmonics can be neglected for $\beta_{c} \gg 1$. In this limit the fluctuations $\delta \gamma_{n}(t)$ are small and we may use the expansion

$$
\sin \gamma_{0}(t) \simeq \sin \left(\theta_{0}+\omega t\right)+\cos \left(\theta_{0}+\omega t\right) \delta \gamma_{0}(t)
$$

while for $n \neq 0$ we have

$$
\sin \gamma_{n}(t) \simeq \sin \theta_{n}+\cos \theta_{n} \delta \gamma_{n}(t) .
$$

The bias current density $j$ on the l.h.s. of the RSJequations Eq. (33) is time-independent and equal for all junctions, while the quantities on the r.h.s have both time-independent and oscillating components.

Let us discuss the equations for the non-resistive junctions $(n \neq 0)$ first. Here the dc-component is:

$$
j=j_{c} \sin \theta_{n}
$$

This fixes the constant part of the phases in the nonresistive junctions and relates it to the bias current.

For the oscillating part of Eq. (33) one obtains:

$$
0=j_{c} \cos \theta_{n} \delta \gamma_{n}(t)+\sigma \frac{\hbar}{2 e d} \delta \dot{\gamma}_{n}(t)+\dot{D}_{n}(t)
$$

or

$$
0=\bar{\omega}_{p}^{2} \delta \gamma_{n}(t)+\frac{\sigma}{\epsilon_{0}} \delta \dot{\gamma}_{n}(t)+\frac{2 e d}{\hbar \epsilon_{0}} \dot{D}_{n}(t)
$$

with the reduced Josephson plasma frequency

$$
\bar{\omega}_{p}^{2}=\omega_{p}^{2} \sqrt{1-\left(\frac{j}{j_{c}}\right)^{2}} .
$$

Now we discuss the resistive junction at $n=0$. Keeping only the lowest harmonic we find 


$$
\sin \gamma_{0}(t) \simeq \sin \left(\theta_{0}+\omega t\right)+\Re\left(\delta \gamma_{0} e^{i \theta_{0}}\right) .
$$

The dc-component of the RSJ-equation Eq. (33) is therefore given by

$$
j(V)=j_{\mathrm{qp}}(V)+j_{c} \Re\left(\delta \gamma_{0} e^{i \theta_{0}}\right),
$$

where $V$ is the dc-voltage of the resistive junction and $j_{\mathrm{qp}}(V)=\sigma E_{d c}$ is the quasiparticle current density.

For the oscillating part one finds:

$$
0=j_{c} \sin \left(\theta_{0}+\omega t\right)+\sigma \frac{\hbar}{2 e d} \delta \dot{\gamma}_{0}(t)+\dot{D}_{0}(t) .
$$

The two equations (40, 45) can be combined to one inhomogeneous linear differential equation for all $n$

$$
\bar{\omega}_{p}^{2} \delta \gamma_{n}(t)+\frac{\sigma}{\epsilon_{0}} \delta \dot{\gamma}_{n}(t)+\frac{2 e d}{\hbar \epsilon_{0}} \dot{D}_{n}(t)=f_{n}(t)
$$

with

$$
f_{n}(t)= \begin{cases}\bar{\omega}_{p}^{2} \delta \gamma_{0}(t)-\omega_{p}^{2} \sin \left(\theta_{0}+\omega t\right) & \text { for } n=0 \\ 0 & \text { for } n \neq 0 .\end{cases}
$$

Assuming a time dependence of the form $e^{-i \omega t}$ for all oscillating quantities we have

$$
\bar{\omega}_{p}^{2} \delta \gamma_{n}+\frac{-i \omega \sigma}{\epsilon_{0}} \delta \gamma_{n}+\frac{2 e d}{\hbar \epsilon_{0}}(-i \omega) D_{n}=f_{n}
$$

with

$$
f_{n}= \begin{cases}\bar{\omega}_{p}^{2} \delta \gamma_{0}-\frac{i \omega_{p}^{2}}{2} e^{-i \theta_{0}} & \text { for } n=0 \\ 0 & \text { for } n \neq 0 .\end{cases}
$$

In order to incorporate the non-local dependence of the polarisation on the electric fields in different barriers a spatial Fourier representation of the form

$$
\delta \gamma_{n}=\frac{1}{N_{z}} \sum_{q_{z}} \gamma\left(q_{z}\right) e^{i q_{z} z_{n}}
$$

is introduced. Using the relation

$$
D\left(q_{z}\right)=\epsilon_{0} \epsilon_{\mathrm{ph}}^{L}\left(q_{z}, \omega\right) E\left(q_{z}\right)=\frac{\epsilon_{0} \hbar}{2 e d} \epsilon_{\mathrm{ph}}^{L}\left(q_{z}, \omega\right)(-i \omega) \gamma\left(q_{z}\right)
$$

in Eq. (48) yields

$$
G^{-1}\left(q_{z}, \omega\right) \gamma\left(q_{z}\right)=f_{0}
$$

with

$$
G^{-1}\left(q_{z}, \omega\right)=\bar{\omega}_{p}^{2}-i \omega \frac{\sigma}{\epsilon_{0}}-\omega^{2} \epsilon_{\mathrm{ph}}^{L}\left(q_{z}, \omega\right) .
$$

For the phase oscillation in the resistive junction follows

$$
\delta \gamma_{0}=\frac{1}{N_{z}} \sum_{q_{z}} \gamma\left(q_{z}\right)=g(\omega) f_{0}
$$

with

$$
g(\omega)=\frac{1}{N_{z}} \sum_{q_{z}} G\left(q_{z}, \omega\right)
$$

Solving for $\delta \gamma_{0}$

$$
\delta \gamma_{0}=\frac{1}{2} \frac{-i \omega_{p}^{2}}{g^{-1}(\omega)-\bar{\omega}_{p}^{2}} e^{-i \theta_{0}}
$$

we obtain

$$
\Re\left(\delta \gamma_{0} e^{i \theta_{0}}\right)=\frac{1}{2} \Im \frac{\omega_{p}^{2}}{g^{-1}(\omega)-\bar{\omega}_{p}^{2}} .
$$

From this finally the following expression for the dccurrent density as function of the dc voltage is obtained:

$$
\begin{aligned}
j(V) & =j_{\mathrm{qp}}(V)-\frac{j_{c}}{2} \frac{\omega_{p}^{2}}{\omega^{2}} \Im \frac{1}{\tilde{\epsilon}(\omega)} \\
& =j_{\mathrm{qp}}(V)+\frac{j_{c}}{2} \frac{\omega_{p}^{2}}{\omega^{2}} \frac{\tilde{\epsilon}_{2}(\omega)}{\tilde{\epsilon}_{1}^{2}(\omega)+\tilde{\epsilon}_{2}^{2}(\omega)} .
\end{aligned}
$$

Here $\tilde{\epsilon}(\omega)$ is a modified dielectric function

$$
\tilde{\epsilon}(\omega)=\bar{\epsilon}_{\mathrm{ph}}(\omega)+\frac{i \sigma}{\epsilon_{0} \omega}
$$

where

$$
\bar{\epsilon}_{\mathrm{ph}}(\omega)=J^{-1}(\omega)+\frac{\bar{\omega}_{p}^{2}}{\omega^{2}}-\frac{i \sigma}{\epsilon_{0} \omega}
$$

and

$$
J(\omega)=\frac{1}{N_{z}} \sum_{q_{z}}\left[\epsilon_{\mathrm{ph}}^{L}\left(q_{z}, \omega\right)-\frac{\bar{\omega}_{p}^{2}}{\omega^{2}}+\frac{i \sigma}{\epsilon_{0} \omega}\right]^{-1} .
$$

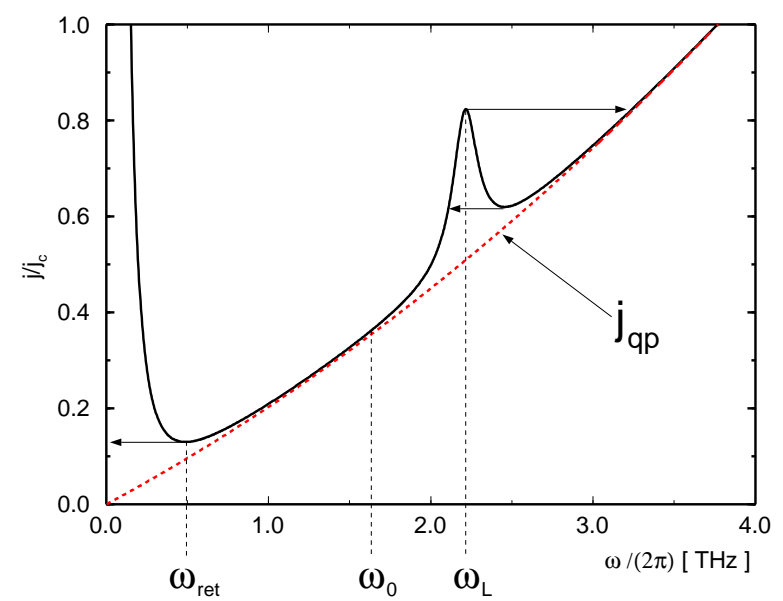

FIG. 2. Analytical $I$ - $V$-curve for one resistive junction with one phonon resonance at $\omega=\omega_{L}$. The arrows mark the hysteretic jumps found in current-biased experiments and numerical simulations. 
This expression describes the dc-current density as function of the dc-voltage $V=\hbar \omega /(2 e)$. It has a maximum for frequencies $\omega$ where the real part $\tilde{\epsilon}(\omega)$ vanishes, i.e. for longitudinal phonon frequencies.

This can easily be seen if we consider the special case of one phonon mode without dispersion. Then $\bar{\epsilon}_{\mathrm{ph}}(\omega)=$ $\epsilon_{\mathrm{ph}}^{L}(\omega)$, which is of the form Eq. (32). The corresponding $I$-V -curve is shown in Fig. 2, which is calculated with the dielectric function

$$
\epsilon_{\mathrm{ph}}^{L}(\omega)=1+\frac{\Omega^{2}}{\omega_{L}^{2}-\Omega^{2}-\omega^{2}-i \omega \rho} .
$$

Here an additional damping $\rho$ of the phonon has been introduced in Eq. (32) in order to simulate the energy transfer into other junctions due the coupling of the ions. A peak in the $I$ - $V$-curve appears at $\omega=\omega_{L}$. The width of the peak is determined by this damping together with the quasiparticle conductivity. The deviation from the quasiparticle current density vanishes at the pole of $\epsilon_{\mathrm{ph}}^{L}(\omega)$ at $\omega_{0}=\sqrt{\omega_{L}^{2}-\Omega^{2}}$. The rise at low voltages is due to the plasma resonance. For $\beta_{c} \gg 1$ the minimum of the $I$ $V$-curve is at $\omega_{\text {ret }} \simeq(3 / 2)^{1 / 4} \omega_{p} / \sqrt{\epsilon_{\mathrm{ph}}^{L}\left(\omega_{p}\right)}$. In currentbiased experiments and corresponding numerical simulations the parts with negative differential conductivity are skipped hysteretically as indicated in the figure.

Note that the denominator in the function $J(\omega)$ Eq. (61) is the total $q_{z^{-}}$and $\omega$-dependent longitudinal dielectric function of the coupled system of phonons and conduction electrons,

$$
\epsilon_{\mathrm{tot}}^{L}\left(q_{z}, \omega\right)=\epsilon_{\mathrm{ph}}^{L}\left(q_{z}, \omega\right)-\frac{\bar{\omega}_{p}^{2}}{\omega^{2}}+\frac{i \sigma}{\epsilon_{0} \omega} .
$$

Zeros of the real part of this function describe longitudinal collective modes in the system. On the other hand the resonances in the $I-V$ curve appear at the bare longitudinal phonon frequency in the case of a narrow phonon band. The summation over $q_{z}$ in Eq. (61) leads to an effective damping of the resonances which is proportional to the phonon dispersion. The physical origin is the loss of energy by phonons from the resistive junction to the neighboring junctions.

The result for the current-voltage characteristic can be generalised to the case of several junctions being in the resistive state, if we assume that all junctions oscillate with the same frequency $\omega$. Denoting the subset of indices for the resistive junctions by $I$ then for $i \in I$ we obtain (for a derivation see the appendix):

$$
\begin{aligned}
j(V) & =j_{\mathrm{qp}}\left(\frac{\hbar \omega}{2 e}\right) \\
& -\frac{j_{c}}{2} \frac{\omega_{p}^{2}}{\omega^{2}} \Im \sum_{k \in I} e^{i \theta_{i}}\left[\bar{\epsilon}(i, k, \omega)+\frac{i \sigma}{\epsilon_{0} \omega} \delta_{i, k}\right]^{-1} e^{-i \theta_{k}}
\end{aligned}
$$

The dielectric function $\bar{\epsilon}(i, k, \omega)$ is defined by

$$
\begin{aligned}
\bar{\epsilon}(i, k, \omega) & :=\left[\frac{1}{N_{z}} \sum_{q_{z}} \frac{e^{i q_{z}\left(z_{i}-z_{k}\right)}}{\epsilon_{\mathrm{ph}}^{L}\left(q_{z}, \omega\right)-\frac{\bar{\omega}_{p}^{2}}{\omega^{2}}+\frac{i \sigma}{\epsilon_{0} \omega}}\right]^{-1} \\
& +\left(\frac{\bar{\omega}_{p}^{2}}{\omega^{2}}-\frac{i \sigma}{\epsilon_{0} \omega}\right) \delta_{i, k}
\end{aligned}
$$

The terms in brackets in Eqs. 64, 65) are understood as matrix inversions. The dc-voltage $V$ is obtained, if one multiplies $\hbar \omega /(2 e)$ by the number of resistive junctions. Note that the r.h.s. of Eq. (64) depends on the layer index $i \in I$, while the l.h.s. is equal for each junction. From this equality the (relative) phases $\theta_{i}$ in the different junctions can in principle be determined, which in turn provides an analytical expression for the $I-V$-curve.

In the case of two resistive junctions exactly two solutions exist with $\theta_{i}=\theta_{j}$ and $\theta_{i}=\theta_{j}+\pi$, respectively. In the general case several different solutions are found. The stability of these solutions will be checked by a comparison with a direct numerical integration of the coupled equations of motions in the following section. It turns out, that those analytical solutions are most stable where the phases $\theta_{i}$ of the oscillating Josephson junctions fit best to the pattern of lattice vibrations with the given frequency $\omega$.

\section{A SIMPLE EXAMPLE}

The theory in the preceding sections is developed for general lattice dynamical models and is valid within the assumptions we have made for the superconducting properties: we treat only single-layer systems assuming a homogeneous conduction electron charge distribution along the layers. An extension of the theory to more realistic systems is in principle possible, but requires to introduce the charge susceptibility of conduction electrons in the superconducting state and a generalisation of the Josephson theory to multilayer systems. But for this more details of the electronic properties are required than are currently known about these materials. In addition to this, it is not possible to compare theoretical results for the $I$ - $V$-curves with experiments in detail, as reliable lattice dynamical calculations for the strongly anisotropic systems BSCCO and TBCCO with two and three layers and variable oxigen content are not yet available.

In the following we consequently only want to illustrate the main features of our theory in a simple toy model, which reflects some basic aspects of the real system. One of the main lattice dynamical property of these systems is certainly the existence of a longitudinal acoustical and (several) flat longitudinal optical bands which result from movements of groups of ions in the barrier against ions in the $\mathrm{CuO}_{2}$-planes in the c-direction.18 20 Such modes we simulate by the most simple lattice dynamical model consisting of two kinds of ions with ionic charges $Z_{l}$, $Z_{b}=-Z_{l}$ and masses $M_{l}, M_{b}$. The first kind $(\kappa=l)$ is placed on the superconducting layers, the second kind $(\kappa=b)$ in the middle of the barrier. The motion of ions 
in the c-direction which is assumed to be uniform along the layers is approximated by a two-atomic chain-model with nearest-neighbor interactions in the c-direction:

$$
\begin{aligned}
& M_{l} \ddot{u}\left(\begin{array}{c}
n \\
l
\end{array}\right)-f\left(u\left(\begin{array}{c}
n \\
b
\end{array}\right)+u\left(\begin{array}{c}
n-1 \\
b
\end{array}\right)-2 u\left(\begin{array}{c}
n \\
l
\end{array}\right)\right)=Z_{l} E^{\rho}\left(\begin{array}{c}
n \\
l
\end{array}\right) \\
& M_{b} \ddot{u}\left(\begin{array}{c}
n \\
b
\end{array}\right)-f\left(u\left(\begin{array}{c}
n+1 \\
l
\end{array}\right)+u\left(\begin{array}{c}
n \\
l
\end{array}\right)-2 u\left(\begin{array}{c}
n \\
b
\end{array}\right)\right)=Z_{b} E^{\rho}\left(\begin{array}{c}
n \\
b
\end{array}\right) .
\end{aligned}
$$

By choosing the masses very different a narrow optical band can be simulated. From a diagonalisation of the dynamical matrix given by Eq. (66) the well-known eigenfrequencies $\omega\left(q_{z} \lambda\right)$ of the two-atomic chain are obtained. With help of the eigenvectors the oscillator strengths defined in Eq. (29) are calculated, which are needed for the longitudinal dielectric function Eq. (31).

The driving field on the r.h.s. of Eq. (66) is the field set-up by the conduction electron charges on the superconducting layers, which can be expressed by the (constant) field $E_{n}^{\rho}$ in the barrier between layers $n$ and $n+1$ with the help of Eq. (18). The latter can be expressed by the average total electric field $E_{n}$ in the barrier:

$$
E_{n}^{\rho}=E_{n}+\frac{1}{\epsilon_{0}} P_{n}
$$

where the polarisation Eq. (25) is given by:

$$
P_{n}=\frac{e}{v_{c}}\left(Z_{b} u\left(\begin{array}{c}
n \\
b
\end{array}\right)+\frac{1}{2} Z_{l}\left(u\left(\begin{array}{c}
n \\
l
\end{array}\right)+u\left(\begin{array}{c}
n+1 \\
l
\end{array}\right)\right)\right) .
$$

These equations for the motion of lattice displacements have to be supplemented by the extended RSJ-equations Eq. (33):

$$
j=j_{c} \sin \gamma_{n}(t)+\sigma \frac{\hbar}{2 e d} \dot{\gamma}_{n}(t)+\epsilon_{0} \frac{\hbar}{2 e d} \ddot{\gamma}_{n}(t)+\dot{P}_{n}(t) .
$$

This model is used to calculate the $I$ - $V$-characteristics in two ways: 1 . The $I$ - $V$-curves are calculated analytically using the results Eq. (64) obtained by the Green's function method. Thus the peaks due to the phonon resonances are obtained. 2. The coupled set of RSJ equations Eq. (69) and phonon equations Eqs. (66, 68) are integrated numerically by a Runge-Kutta method for a finite stack of Josephson junctions. Changing the biascurrent gradually allows to follow the $I-V$-curves as in the current-biased experimental situation and to reproduce the hysteretic behaviour in particular.

We start with the discussion of the first branch of the $I$ - $V$-curve, where one junction is in the resistive state.

\section{A. One resistive junction}

Quite generally the $I$ - $V$-curve is expected to have peaks at the van Hove singularities of the phonon dispersion. Details, however, depend on the oscillator strength defined by Eq. (29) which enters the longitudinal dielectric function Eq. (31). In particular at the edge of the Brillouin zone for $q_{z}=\pi / d$ only the motion of ions within the barrier contribute to the oscillator strength, the ions on the superconducting layers are inactive due to the factor $1+\exp \left(i q_{z} d\right)$ in Eq. (21). These features will be illustrated in the following.
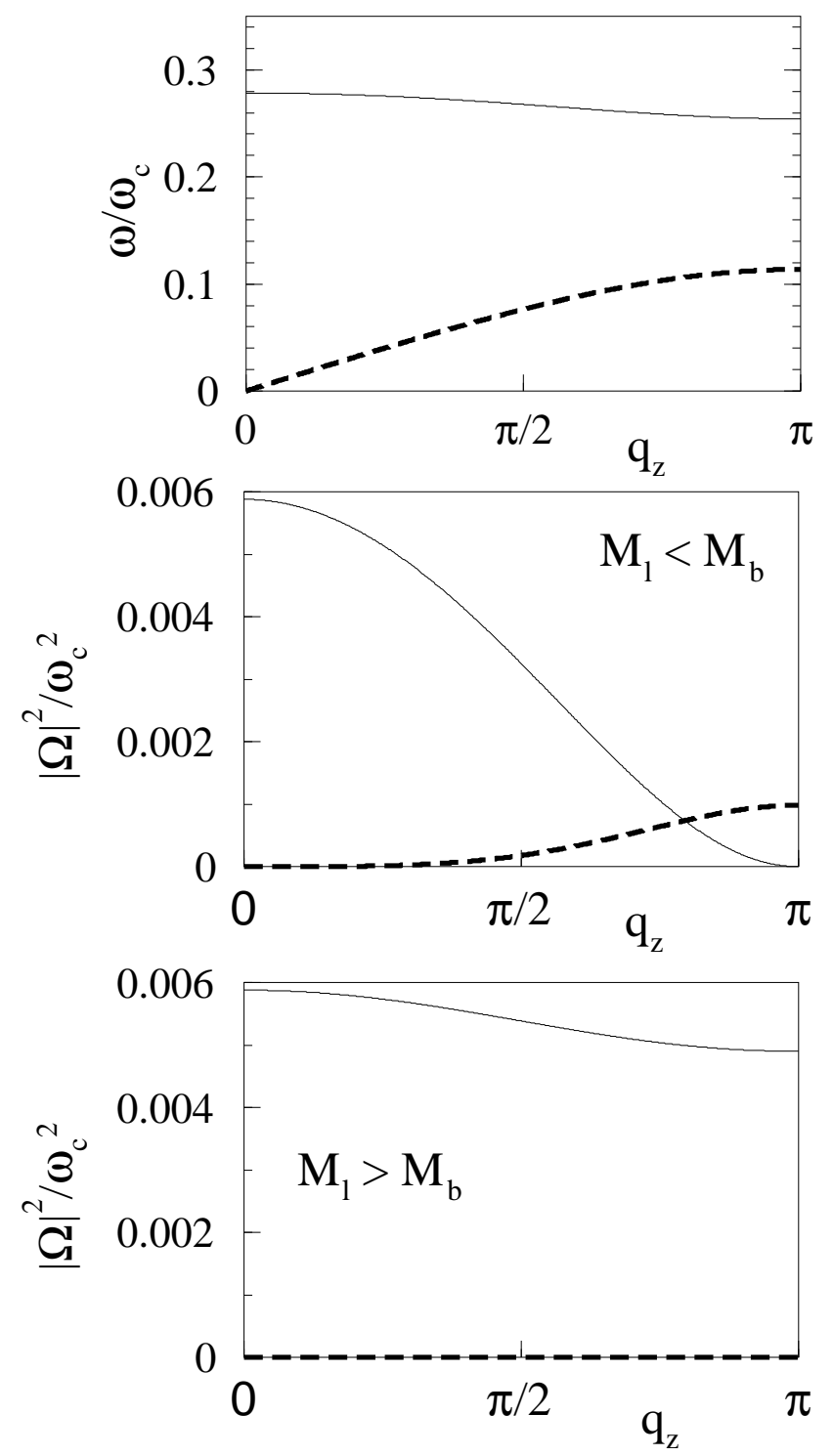

FIG. 3. Dispersion and oscillator strengths for the two-atomic chain model. Shown are the oscillator strengths $\left|\Omega\left(q_{z} \lambda\right)\right|^{2}$ for the acoustical branch (dashed curve) and the optical branch (solid curve) and for the two cases of the heavy ions on the superconducting layer $\left(M_{l}>M_{b}\right)$ and in the barrier $\left(M_{l}<M_{b}\right)$.

For the lattice dynamical model introduced above at $q_{z}=\pi / d$ only one type of particles is moving due to symmetry: In the acoustical branch the heavier ion, in the optical branch the lighter ion is moving. If the heavier ion is on the superconducting layers $\left(M_{l}>M_{b}\right)$ the oscillator strength vanishes at the end of the acoustic branch (see Fig. 3), and peaks are expected to appear in the $I-V$-curve at the two van-Hove singularities of the optical branch. On the other hand, if the lighter ion is on 
the superconducting layers $\left(M_{l}<M_{b}\right)$ then the oscillator strength vanishes at the end of the optical branch, and peaks are expected at $q_{z}=\pi / d$ from the acoustical branch and at $q_{z}=0$ from the optical branch.
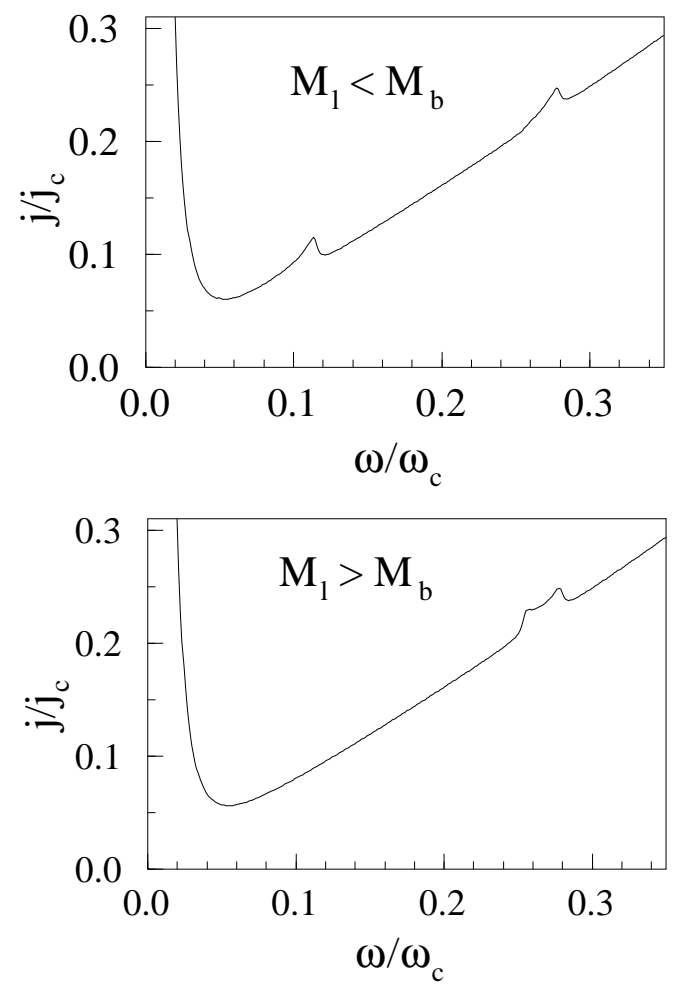

FIG. 4. $I$ - $V$-curves for one resistive junction with subgap-structures due to acoustical and optical phonons.

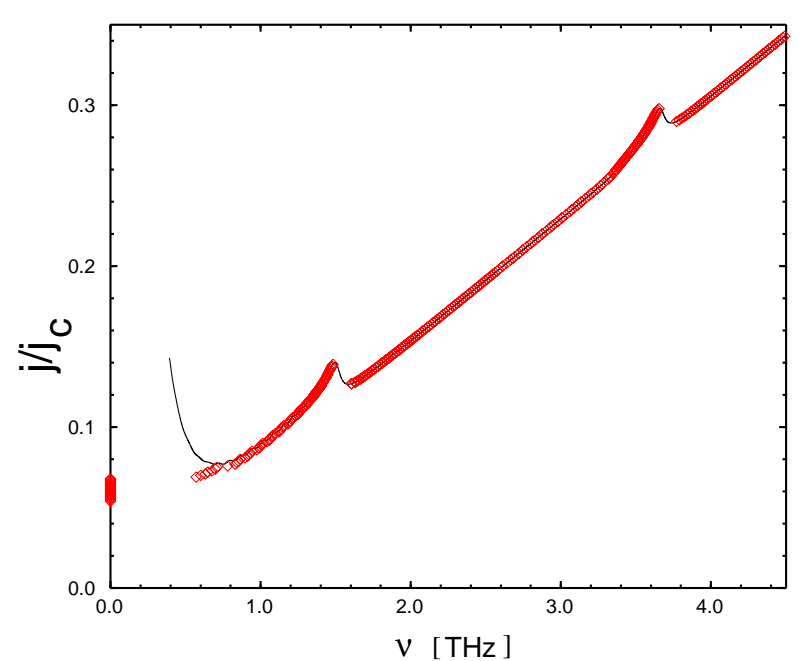

FIG. 5. Comparison between analytical (solid line) and numerical results (rhombs) for the $I-V$-curve of one resistive junction with subgap-structures due to acoustical and optical phonons for $M_{l}<M_{b}$.

This is illustrated in Fig. 4 where we have plotted results for the $I-V$-curve of the first branch in the two cases $M_{l}<M_{b}$ and $M_{l}>M_{b}$. In our model the phonon dispersion is fixed by the values of $\omega_{\mathrm{LO}}^{2}\left(q_{z}=\right.$ $0)=2 f\left(1 / M_{l}+1 / M_{b}\right)$ and the mass ratio $M_{l} / M_{b}$. A measure for the oscillator strength is the quantity $\Omega_{l, b}^{2}:=Z^{2} /\left(M_{l, b} \epsilon_{0} v_{c}\right)$. In Fig. 囵 we have used the following parameters: $\beta_{c}=\omega_{c}^{2} / \omega_{J}^{2}=375, M_{l} / M_{b}=0.2$ and $M_{b} / M_{l}=0.2$ respectively. The phonon frequencies are normalised to $\omega_{c}$ and are given by $\omega_{\mathrm{LO}}\left(q_{z}=0\right)=0.28 \omega_{c}$. The oscillator strength is given by $\omega_{c}^{2} / \Omega_{l}^{2}=200$ for $M_{l}<M_{b}$ and $\omega_{c}^{2} / \Omega_{b}^{2}=200$ for $M_{l}>M_{b}$. The corresponding phonon dispersion and the oscillator strengths are shown in Fig. 3. The values of the parameters $\beta_{c}$, $\omega_{L O} / \omega_{c}$ are adapted to TBCCO, also the chosen value $|\Omega|^{2} / \omega_{0}^{2}=0.08$ for the oscillator strength compares well to the experimental estimate $\approx 0.1311$. The mass-ratio is chosen to obtain a sufficiently flat optical branch (cf. the band structure in Ref. 19).

For $M_{l}<M_{b}$ the analytical results for the $I$ - $V$-curve in Fig. 4 show phonon peaks at the van Hove singularity of the acoustic branch at $q_{z}=\pi / d$ and at the van Hove singularity at $q_{z}=0$ of the optical branch, the resonance at the van Hove singularity at $q_{z}=\pi / d$ of the optical branch is suppressed. For $M_{l}>M_{b}$ only structures due to the two van Hove singularities of the optical branch appear. In both cases the increase of the $I$ - $V$-curve at low frequencies indicates the Josephson plasma frequency. The numerical results shown in Fig. 5 for $M_{l}<M_{b}$ (here $\omega_{c}$ $=13.1 \mathrm{THz}$ ) follow the analytical results in the regions of positive differential resistance perfectly, and otherwise show the hysteretic behaviour as seen in experiments. At low values of $j / j_{c}$ the $I$ - $V$-curve switches back to the superconducing state of the junction.

\section{B. Two resistive junctions}

Another important effect of the coupling between Josephson oscillations and phonons is the synchronisation (phase-locking) of Josephson oscillations in different resistive junctions, which would be absent without phonons in short junctions, which are homogeneous parallel to the layers. we want to illustrate this for the case of two resistive junctions coupled by one narrow optical phonon branch.

The second branch of the $I$ - $V$-curve which corresponds to two resisitve junctions has a rather complex structure already for one phonon band. This is shown schematically in Fig. 6. If we denote the two dynamical states of the first branch by $a$ and $b$, then on the second branch with two resistive junctions either both junctions are in state $a$ (label $a a$ ), both junctions are in state $b$ (label $b b$ ) or one junction is in state $a$ while the other junction is in state $b$ (label $a b$ ). Note that in the latter case the oscillation frequencies of the two junctions are different. In the case of well separated resistive junctions the voltages of a structure for a given bias current are determined by $\omega_{a a}=2 \omega_{a}, \omega_{b b}=2 \omega_{b}, \omega_{a b}=\omega_{a}+\omega_{b}$. This is no longer true, if the resistive junctions are close to each other and 
interact via phonons. Then the voltages in the second branch are slightly lower.

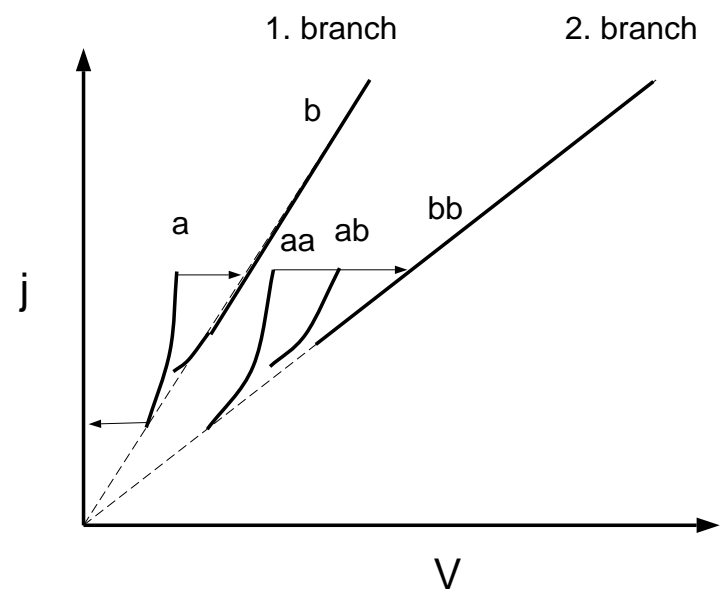

FIG. 6. Schematic plot of the first and second branch of the $I-V$-curve with subgap structures due to one phonon.

In the case of two resistive junctions $i$ and $j$ two solutions of Eq. (64) exist with phase differences $\theta_{i}=\theta_{j}$ and $\theta_{i}=\theta_{j}+\pi$, corresponding to in-phase and out-of-phase Josephson oscillations, respectively. Inserting these results in Eq. (64) two different $I-V$-curves can be calculated. Note that this formula applies only for the states $a a$ and $b b$, because in the derivation we have assumed that the two junctions oscillate with the same frequency. A similar formula can also be derived for the state $a b$. In that case the phases are meaningless because the two junctions oscillate with different frequencies and are essentially decoupled.

In Fig. 7 we show examples calculated for a narrow optical band with $M_{l} / M_{b}=10$ and the light ion oscillating in the barrier. Here the different analytical solutions are shown together with numerical results for neighboring resistive junctions, $j=i+1$ (a), and two resistive junctions separated by 1 or 2 superconducting junctions, $j=i+2(\mathrm{~b}), j=i+3(\mathrm{c})$.

It is plausible that in the case of neighboring resistive junctions out-of-phase Josephson oscillations $\left(\theta_{i}=\theta_{j}+\right.$ $\pi$ ) favor a coupling to phonons at the edge $q_{z}=\pi / d$ of the Brillouin zone, while the coupling of in-phase oscillations $\left(\theta_{i}=\theta_{j}\right)$ is strongest for zone-center phonons at $q_{z}=0$. This is shown in Fig. 7a, where the $I$ - $V$-curve for the inphase solution shows a peak at $\omega\left(q_{z}=0\right)$, while for the out-of-phase solution the current-maximum is at $\omega\left(q_{z}=\right.$ $\pi / d)$.

It can be seen that the numerical results in the dynamical state $a a$ follow one of the analytical solutions before a hysteretic switch into state $b b$ occurs (outside the figure). This is verified in Fig. I a where the numerical $I$ - $V$-curve follows the analytical curve for $\theta_{i}=\theta_{i+1}+\pi$. In Fig. $7 \mathrm{~b}$ the in-phase solution with $\theta_{i}=\theta_{i+2}$ has maxima for voltages corresponding to frequencies of optical phonons at both $q_{z}=\pi / d$ and $q_{z}=0$, while the out-of-phase solution has a broad maximum in the middle of the Brillouin zone. The numerical results follow the in-phase solution. Fig. 17c shows results for $j=i+3$.
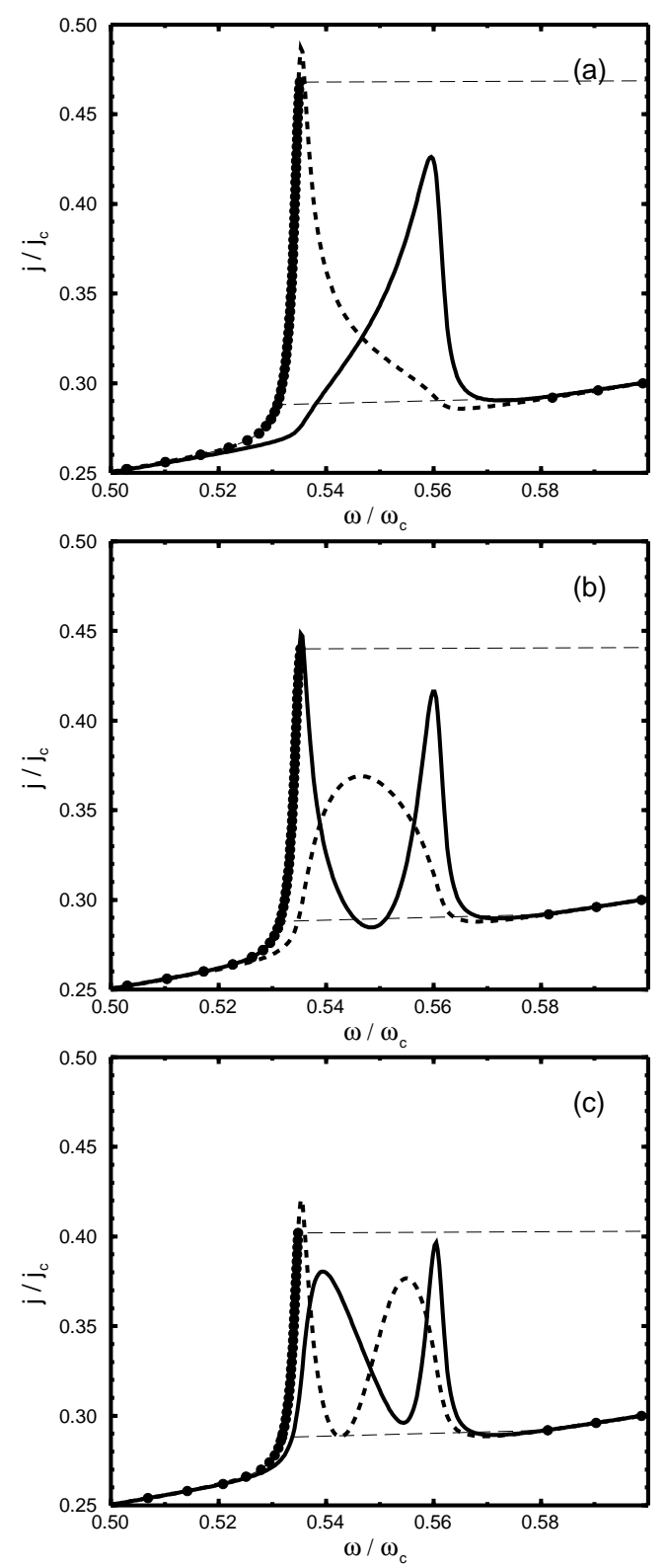

FIG. 7. $I$ - $V$-curve for two resistive junctions separated by 0 (a), 1 (b), 2 (c) superconducting junctions. Shown are analytical $I$ - $V$-curves calculated for a narrow optical band for the in-phase, $\theta_{j}-\theta_{i}=0$ (solid line) and the out-of-phase, $\theta_{j}-\theta_{i}=\pi$ (dashed line) solution together with numerical results (dots).

\section{Several resistive junctions}

The results obtained for two resistive junctions show that the peak position in the $I$ - $V$-curve depends only slightly on the distance between the resistive junctions. More important is the fact that phonons are able to synchronise the phases of Josephson oscillations in different 
resistive junctions. This is important for the use of such systems in high-frequency applications. We have checked this numerically for the case of a block of many resistive junctions. For frequencies $\omega$ close to a phonon eigenfrequency $\omega\left(q_{z}=\pi / d\right)$ at the edge of the Brioullin zone the Josephson field oscillations in neighboring junctions differ by a value close to $\pi$. For frequencies close to $\omega\left(q_{z}=0\right)$ near the $\Gamma$-point the Josephson oscillations are nearly in phase. These phase-locked dynamical states are reached from arbitrary initial conditions for the phases. There is also a synchronisation of Josephson oscillations for frequencies far away from phonon resonances. We did not yet investigate the stability of these states systematically but we expect these to be less stable than at frequencies close to a phonon resonance.

\section{EXPERIMENTAL RESULTS}

Recently the explanation of the subgap resonances in Refs. 3 3,8 8 with the phonon coupling mechanism presented here could be well confirmed by Raman measurements on the same samplest21 and infrared reflectivity experiments with grazing incidence, 11,22 where the latter allows to determine both longitudinal and transverse modes (see Table I). Small deviations of the order of $\sim 5-10 \%$ may be attributed to the fact that in optical experiments and in the intrinsic Josephson effect different averages over $\vec{q}$ of the dielectric functions are relevant. Note that in our theory modes which are Raman active at $q_{z}=0$ may couple to intrinsic Josephson_oscillations also for $q_{z} \neq 0$. Earlier experimental data, 10 which are obtained from polycrystalline samples, show the same qualitative behaviour, but differ in detail.

With the help of rigid-ion 23 and shell-model calculations 1820 some of the more pronounced structures can be connected with certain elongation patterns of the ions in the unit cell. For example, the peak in the $I-V$ curve at $4.64 \mathrm{THz}$ in $\mathrm{Tl}_{2} \mathrm{Ba}_{2} \mathrm{Ca}_{2} \mathrm{Cu}_{3} \mathrm{O}_{10}$ seems to be due to a $(\mathrm{Cu}, \mathrm{Ba})$-mode.

The qualitative features of the subgap resonances have already been explained with the help of the phonon interpretation in Ref. 8: The position of the resonance is independent on temperature, magnetic field and the geometry of the probe, while the intensity of the structure varies $\sim j_{c}^{2}$ with the critical current density $j_{c}(T, B)$. Also the behaviour of the position and intensity of the structures in external pressure are in agreement with the phonon explanation and formula (11).

One of the main qualitative features of the general theory with dispersive phonon bands, which goes beyond the local oscillator model used in Refs. 6,8, is the possibility to describe resonances at van-Hove singularities, which appear e.g. at the upper band edge of the acoustical phonon band.

This might be an explanation for a resonance seen in Ref. 5 at $3.2 \mathrm{mV}(\hat{=} 1.54 \mathrm{THz})$ in the $I-V$-characteristic of $\mathrm{Tl}_{2} \mathrm{Ba}_{2} \mathrm{Ca}_{2} \mathrm{Cu}_{3} \mathrm{O}_{10}$, because the same frequency is expected by lattice dynamical calculations 19 for the upper edge of the acoustical band, and there are no optical phonon bands in this low frequency range. Fig. 8 shows a fit of a $I$ - $V$-curve calculated with the two-atomic chain model (and some additional damping) to the experimental results from Ref. 5 .

Similarily in BSCCO the upper edge of the longitudinal acoustical phonon band, which has heen detected in inelastic neutron scattering at $5 \mathrm{meV} 24$, might correspond to a less pronounced phonon resonance in the intrinsic Josephson effect at $V_{\mathrm{sg}}=4.9 \mathrm{mV}(\hat{=} 2.38 \mathrm{THz})$, which seems to be invisible in optical experiments. Nevertheless at the present time this interpretation is not yet fully conclusive, as the instrumental resolution of $0.65 \mathrm{meV}$ in the neutron scattering experiment is still rather large compared with the accuracy in the measurement of the $I-V$-curve.

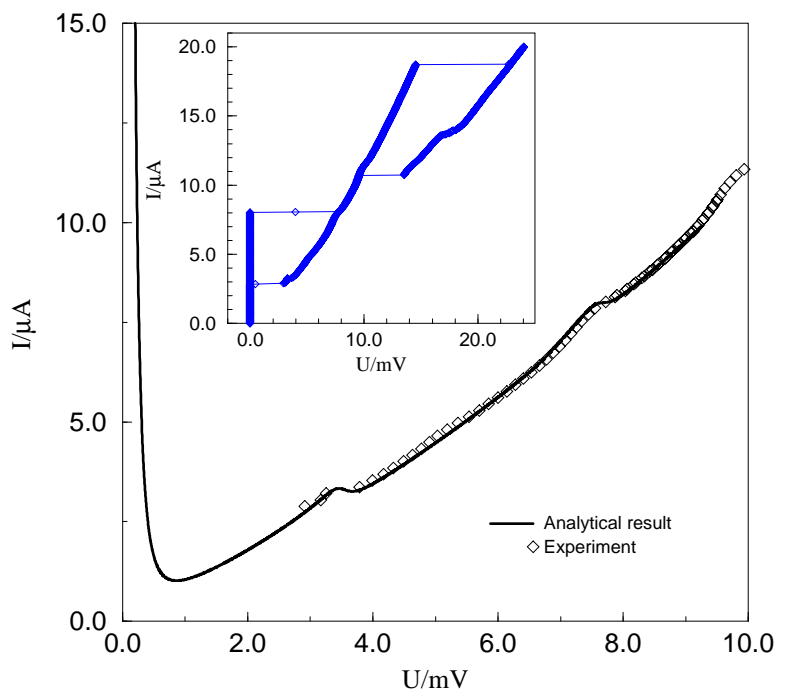

FIG. 8. Fit of the experimental $I$ - $V$-curve in $\mathrm{Tl}_{2} \mathrm{Ba}_{2} \mathrm{Ca}_{2} \mathrm{Cu}_{3} \mathrm{O}_{10}$ from Ref. $\mathrm{B}$ near the subgap resonances at the band edge of the acoustical branch (at $1.5 \mathrm{THz}$ ) and an optical branch with the help of the a two-atomic chain model. The inset shows experimental results over a wider frequency range.

Also the effect of the two van-Hove singularities at the optical band edges on the $I-V$-curve as discussed above might have been seen in the satellite structures at 5.17 $\mathrm{THz}(107 \mathrm{mV})$ and $5.6(11.6 \mathrm{mV})$ in the $I-V$-curve of BSCCOU. This interpretation is further supported by the theoretical prediction of the bandwidth $\sim 0.3 \mathrm{THz}$ and the fact that the assignment of no other phonon mode is plausible. 
TABLE I. Comparison of the frequencies $f_{\mathrm{sg}}=\frac{h}{2 e} V_{\mathrm{dc}}$ (in $\mathrm{THz}$ ) of the most pronounced subgap resonances in Ref. 8 and of infrared- and Raman active modes in $\mathrm{Bi}_{2} \mathrm{Sr}_{2} \mathrm{CaCu}_{2} \mathrm{O}_{8}$ and $\mathrm{Tl}_{2} \mathrm{Ba}_{2} \mathrm{Ca}_{2} \mathrm{Cu}_{n} \mathrm{O}_{2 n+4}$.

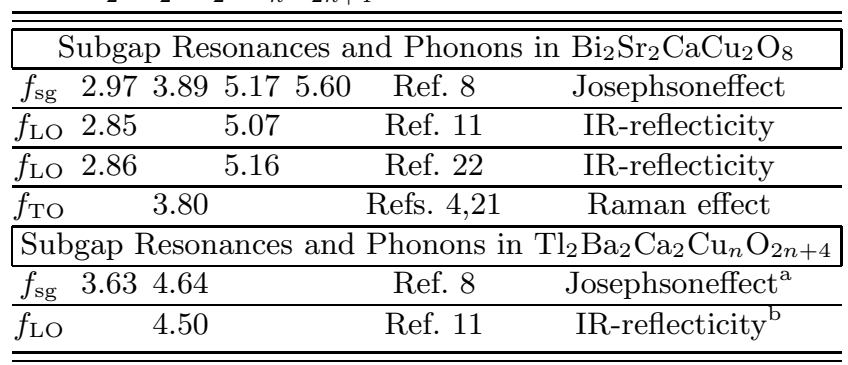

${ }^{\mathrm{a}} \mathrm{Tl}_{2} \mathrm{Ba}_{2} \mathrm{Ca}_{2} \mathrm{Cu}_{3} \mathrm{O}_{10}$

${ }^{\mathrm{b}} \mathrm{Tl}_{2} \mathrm{Ba}_{2} \mathrm{Ca}_{2} \mathrm{Cu}_{2} \mathrm{O}_{8}$

\section{CONCLUSIONS AND OUTLOOK}

In this paper we have developed a microscopic theory for the coupling between Josephson oscillations and phonons in intrinsic Josephson systems like the highly anisotropic cuprate superconductors. We determined the precise form of the longitudinal dielectric function Eq. (31) describing this coupling and obtained analytical results Eqs. (58,64) for the $I$ - $V$-curve for one and several resistive junctions. The principle features and selection rules for phonon resonances in the $I-V$-curves are illustrated with help of a simple lattice dynamical model.

We have shown that not only optical, but also acoustical phonons at the edge of the Brillouin zone couple to Josephson oscillations. This may explain the structure observed in Ref. in the $I$ - $V$-curve occurring at an unusual low voltage (frequency), which is not found in reflectivity experiments, testing transverse optical phonons at $\vec{q}=0$, and in lattice-dynamical calculations for infrared active phonons at $\vec{q}=0$. A weaksatellite-structure observed in the $I$ - $V$-curve of BSCCOI may be due to a double resonance from the two van-Hove singularites of an optical branch.

The analytical results are compared with results from a numerical integration of the coupled equations of motion for the Josephson oscillations and phonons. For this purpose a simplified lattice dynamical model has been used with one acoustical and one optical branch. It is found that in the limit of large values of the McCumber parameter the numerical results follow closely the analytical solutions with the following exceptions: 1. Using a gradual change of the bias-current, regions of the $I-V$ curve with negative differential conductivity are skipped as is observed in current-biased experiments. 2. In the case of several resistive junctions, where several analytical solutions are obtained, the numerical result follows only one of the analytical solutions. The stability of the different analytical solutions is currently investigated. It seems to be that the solution which gives a minimum for the interaction energy between polarisation and the electric field generated by the Josephson oscillations at a given frequency is most stable. The phonons thus serve to synchronise the Josephson oscillations in different resistive layers, which is important for the application of such systems as high-frequency devices.

In this paper we have considered only the case of a current distribution in the c-direction which is homogeneous along the layers. We neglected all magnetic field effects assuming that all quantities are uniform along the layers. For real systems this is an artificial approximation, because the current induced magnetic field can never be avoided completely. Nevertheless, we argue that this is a valid approximation for the intrinsic Josephson systems forming a mesa structure of about fifty layers with a width of several $\mu$ and a thickness which is much smaller. Such a junction is still short with respect to the length $\lambda_{J}=c / \omega_{p}$ describing the variation of the phase-difference along the layers (in order to avoid completely the spontaneous formation of vortices, which have a smaller diameter, the mesa width should not exceed 1-2 $\mu$ ). Therefore the treatment of the superconducting layers as metal sheets with a uniform charge distribution, the creation of uniform polarisation fields and the neglect of $q_{\|}$in the calculation of the longitudinal dielectric function is justified for the systems investigated. This will be different in the case of longer junctions and strong external magnetic field25. In particular the flow of vortices and their interaction with phonons has to be investigated in this case26.

\section{ACKNOWLEDGEMENT}

The authors would like to thank A. Yurgens and A. Tsvetkov for discussions on their experimental results and for providing unpublished data, A. Mayer and D. Strauch for fruitful discussions on lattice dynamical aspects, and P. Müller, R. Kleiner, L. Bulaevskii and A. Bishop for the continuous support of our work on intrinsic Josephson systems. Financial support by the Deutsche Forschungsgemeinschaft, the Bayerische Forschungsstiftung within the research project FORSUPRA, the Studienstiftung des Deutschen Volkes (C.H.) and the US Department of Energy under contract W-7405-ENG-36 (C.H.) is gratefully acknowledged.

\section{APPENDIX A: $I$ - $V$-CURVES FOR SEVERAL RESISTIVE JUNCTIONS}

Quite generally the RSJ-equation for the $\mathrm{n}$-th junction can be written as

$$
\begin{aligned}
j & =j_{c} \sin \gamma_{n}(t)+\sigma \frac{\hbar}{2 e d} \dot{\gamma}_{n}(t) \\
& +\frac{\epsilon_{0} \hbar}{2 e d} \sum_{n^{\prime}} \int_{-\infty}^{\infty} \epsilon_{\mathrm{ph}}^{L}\left(n-n^{\prime}, t-t^{\prime}\right) \ddot{\gamma}_{n^{\prime}}\left(t^{\prime}\right) d t^{\prime} .
\end{aligned}
$$


where $\epsilon_{\mathrm{ph}}^{L}\left(n-n^{\prime}, t\right)$ is the dielectric function Eq. 31 in real space and time domain.

Let us denote the index of a general junction by $n \in$ $\{1 \ldots N\}$ and the subset of indices of resistive junctions by $I$. In the following we assume that oscillations in all junctions are governed by one frequency $\omega$ and the phases can be written as

$$
\gamma_{n}(t)= \begin{cases}\theta_{j}+\omega t+\delta \gamma_{j}(t) & n=j \in I \\ \theta_{n}+\delta \gamma_{n}(t) & \text { else }\end{cases}
$$

with

$$
\delta \gamma_{n}(t)=\delta \gamma_{n} e^{-i \omega t}+\text { c.c. }
$$

Expanding with respect to $\delta \gamma_{n}$ and keeping only the lowest harmonic one finds

$$
\sin \gamma_{n}(t) \simeq \begin{cases}\sin \left(\theta_{j}+\omega t\right)+\Re\left(\delta \gamma_{j} e^{i \theta_{j}}\right) & n=j \in I \\ \sin \theta_{n}+\cos \theta_{n} \delta \gamma_{n}(t) & \text { else. }\end{cases}
$$

Splitting the RSJ-equation (A1) into a dc-part and an oscillating part one obtains for the dc-part:

$$
j= \begin{cases}\sigma E_{d c}+j_{c} \Re\left(\delta \gamma_{j} e^{i \theta_{j}}\right) & \text { for } n=j \in I \\ j_{c} \sin \theta_{n} & \text { else, }\end{cases}
$$

while the oscillating part can be written as $\left(\bar{\omega}_{p}^{2}=\right.$ $\left.\omega_{p}^{2} \sqrt{1-\left(j / j_{c}\right)^{2}}\right)$

$$
\begin{aligned}
\bar{\omega}_{p}^{2} \delta \gamma_{n}(t) & +\frac{\sigma}{\epsilon_{0}} \delta \dot{\gamma}_{n}(t) \\
& +\sum_{n^{\prime}} \int_{-\infty}^{\infty} \epsilon\left(n-n^{\prime}, t-t^{\prime}\right) \delta \ddot{\gamma}_{n^{\prime}}\left(t^{\prime}\right) d t^{\prime}=f_{n}(t)
\end{aligned}
$$

with

$$
f_{n}(t)= \begin{cases}\bar{\omega}_{p}^{2} \delta \gamma_{j}(t)-\omega_{p}^{2} \sin \left(\theta_{j}+\omega t\right) & n=j \in I \\ 0 & \text { else. }\end{cases}
$$

Writing

$$
f_{j}(t)=f_{j} e^{-i \omega t}+c . c .
$$

the amplitude of the driving term for a resistive junction is given by

$$
f_{j}=\bar{\omega}_{p}^{2} \delta \gamma_{j}-i \frac{\omega_{p}^{2}}{2} e^{-i \theta_{j}}
$$

Introducing the spatial Fourier transform

$$
\gamma\left(q_{z}\right)=\frac{1}{N_{z}} \sum_{n=1}^{N} \delta \gamma_{n} e^{-i q_{z} z_{n}}
$$

we obtain

$$
\begin{aligned}
\gamma\left(q_{z}\right) & =G\left(q_{z}, \omega\right) \sum_{j \in I} f_{j} e^{-i q_{z} z_{j}} \\
& =G\left(q_{z}, \omega\right) \sum_{j \in I}\left[\bar{\omega}_{p}^{2} \delta \gamma_{j}-i \frac{\omega_{p}^{2}}{2} e^{-i \theta_{j}}\right] e^{-i q_{z} z_{j}}
\end{aligned}
$$

with the Green's function

$$
G^{-1}\left(q_{z}, \omega\right)=\bar{\omega}_{p}^{2}-i \omega \frac{\sigma}{\epsilon_{0}}-\omega^{2} \epsilon_{\mathrm{ph}}^{L}\left(q_{z}, \omega\right)
$$

of the homogeneous equation.

From this an equation for $\delta \gamma_{i}(i \in I)$ in the resistive junctions is obtained:

$$
\sum_{j \in I}\left(G^{-1}(i, j, \omega)-\bar{\omega}_{p}^{2} \delta_{i, j}\right) \delta \gamma_{j}=-i \frac{\omega_{p}^{2}}{2} e^{-i \theta_{i}}
$$

with

$$
G(i, j, \omega)=\frac{1}{N_{z}} \sum_{q} G(q, \omega) e^{i q\left(z_{i}-z_{j}\right)} .
$$

Using

$$
\bar{\epsilon}_{\mathrm{ph}}(i, k, \omega)=-\frac{1}{\omega^{2}} G^{-1}(i, k, \omega)+\left(\frac{\bar{\omega}_{p}^{2}}{\omega^{2}}-\frac{i \sigma}{\epsilon_{0} \omega}\right) \delta_{i, k}
$$

we get

$$
\delta \gamma_{i}=i \frac{\omega_{p}^{2}}{2 \omega^{2}} \sum_{k \in I}\left[\bar{\epsilon}_{\mathrm{ph}}(i, k, \omega)+\frac{i \sigma}{\epsilon_{0} \omega} \delta_{i, k}\right]^{-1} e^{-i \theta_{k}},
$$

which gives us the dc-component Eq. (A5) of the current density

$$
\begin{aligned}
j(V) & =\sigma E_{d c} \\
& -j_{c} \frac{\omega_{p}^{2}}{2 \omega^{2}} \Im \sum_{k \in I} e^{i \theta_{i}}\left[\bar{\epsilon}_{\mathrm{ph}}(i, k, \omega)+\frac{i \sigma}{\epsilon_{0} \omega} \delta_{i, k}\right]^{-1} e^{-i \theta_{k}} .
\end{aligned}
$$

Finally we want to note that the dielectric function $\bar{\epsilon}_{\mathrm{ph}}(i, k, \omega)$ can also be used to write the RSJ equations in a form where only the phases $\gamma_{i}(t)$, of the resistive junctions $i \in I$ enter:

$$
\begin{aligned}
j=j_{c} \sin \gamma_{i}(t) & +\sigma \frac{\hbar}{2 e d} \dot{\gamma}_{i}(t) \\
& +\frac{\epsilon_{0} \hbar}{2 e d} \sum_{j \in I} \int_{-\infty}^{\infty} \bar{\epsilon}_{\mathrm{ph}}\left(i, j, t-t^{\prime}\right) \ddot{\gamma}_{j}\left(t^{\prime}\right) d t^{\prime}
\end{aligned}
$$

${ }^{1}$ R. Kleiner, F. Steinmeyer, G. Kunkel, P. Müller, Phys. Rev. Lett. 68, 2394 (1992).

${ }^{2}$ R. Kleiner, P. Müller, Phys. Rev. B 49 (1994), 1327.

${ }^{3}$ K. Schlenga, G. Hechtfischer, R. Kleiner, W. Walkenhorst, P. Müller, Phys. Rev. Lett. 76, 4943 (1996).

${ }^{4}$ A. Yurgens, D. Winkler, N. Zavaritsky, T. Claeson, Proceedings of SPIE, Vol 2697, 433 (1996).

${ }^{5}$ P. Seidel, A. Pfuch, U. Hübner, F. Schmidl, H. Schneidewind, T. Ecke, J. Scherbel, Physica C 293, 49 (1997). 
${ }^{6}$ Ch. Helm, Ch. Preis, F. Forsthofer, J. Keller, K. Schlenga, R. Kleiner, P. Müller, Phys. Rev. Lett. 79, 737 (1997).

${ }^{7}$ Ch. Helm, Ch. Preis, F. Forsthofer, J. Keller, K. Schlenga, R. Kleiner, P. Müller, Physica C 293, 60 (1997).

${ }^{8}$ K. Schlenga, R. Kleiner, G. Hechtfischer, M. Mößle, S. Schmitt, P. Müller, Ch. Helm, Ch. Preis, F. Forsthofer, J. Keller, H. L. Johnson, M. Veith, E. Steinbeiß, Phys. Rev. B 57, 14518 (1998).

9 T. Zetterer, M. Franz, J. Schützmann, W. Ose, H.H. Otto, K.F. Renk, Phys. Rev, B 41, 9499 (1990); V.M. Burlakov, S.V. Shulga, J. Keller, K.F. Renk, Physica C 203, 68 (1992) and references therein.

${ }^{10}$ R.G. Buckley, M.P. Staines, D.M. Pooke, T. Stoto, N.E. Flower, Physica C 248, 247 (1995) and references therein.

${ }^{11}$ A. A. Tsvetkov, D. Dulić, D. van der Marel, A. Damascelli, G. A. Kaljushnaia, J. I. Gorina, N. N. Senturina, B. Willemsen, N. N. Kolesnikov, Z. F. Ren, J. H. Wang, A. A. Menovsky, T. T. M. Palstra, Phys. Rev. B 60, 13196 (1999) and references therein.

12 P. Berberich, R. Buemann, H. Kinder, Phys. Rev. Lett. 49, 1500 (1982).

${ }^{13}$ Ya.G. Ponomarev, E.B. Tsokur, M.V. Sudakova, S.N. Tchesnokov, M.A. Lorenz, M.A. Hein, G. Müller, H. Piel, B.A. Animov, Solid State Comm. 111, 513 (1999).

14 T. Koyama, M. Tachiki, Phys. Rev. B 54, 16183 (1996).

${ }^{15}$ Ch. Preis, Ch. Helm, J. Keller, A. Sergeev, R. Kleiner, SPIE Conference Proceedings "Superconducting Superlattices II: Native and Artificial", Vol 3480, 236 (1998).

${ }^{16}$ E.G. Maksimov, P.I. Arseev, N.S. Maslova, Solid State Comm. 111, 391 (1999) .

${ }^{17}$ R. Zeyher, G. Zwicknagl, Solid State Commun. 66, 617 (1988); R. Zeyher, G. Zwicknagl, Z. Phys. B- Condensed Matter 78, 175 (1990).

18 A. D. Kulkarni, J. Prade, F. W. de Wette, W. Kress, U. Schröder, Phys. Rev. B 40, 2642 (1989).

19 A. D. Kulkarni, F. W. de Wette, J. Prade, U. Schröder, W. Kress, Phys. Rev. B 43, 5451 (1991).

${ }^{20}$ J. Prade, A. D. Kulkarni, F. W. de Wette, U. Schröder, W. Kress, Phys. Rev. B 39, 2771 (1989).

${ }^{21}$ A. Yurgens, private communication.

${ }^{22}$ S. Tajima, G. D. Gu, S. Miyamoto, A. Odagawa, N. Koshizuka, Phys. Rev. B 48, 16164 (1993).

${ }^{23}$ C.-S. Jia, P.-Y. Lin, Y. Xiao, X.-W. Jiang, X.-Y. Gou, S. Huo, H. Li, Q.-B. Yang, Physica C 268, 41 (1996).

${ }^{24}$ H. A. Mook, B. C. Chakoumakos, M. Mostoller, A. T. Boothroyd, D. McK. Paul, Phys. Rev. Lett. 69, 2272 (1992).

${ }^{25}$ Ch. Preis, Ch. Helm, K. Schmalzl, Ch. Walter, J. Keller, to appear in Physica C, cond-mat/0001150.

${ }^{26}$ Ch. Preis, K. Schmalzl, Ch. Helm, J. Keller, in preparation. 